\title{
The SLC2 family of facilitated hexose and polyol transporters
}

Received: 4 February 2003 / Accepted: 4 April 2003 / Published online: 16 May 2003

(C) Springer-Verlag 2003

\begin{abstract}
The SLC2 family of glucose and polyol transporters comprises 13 members, the glucose transporters (GLUT) 1-12 and the $\mathrm{H}^{+}$-myo-inositol cotransporter (HMIT). These proteins all contain 12 transmembrane domains with both the amino and carboxy-terminal ends located on the cytoplasmic side of the plasma membrane and a $N$-linked oligosaccharide sidechain located either on the first or fifth extracellular loop. Based on sequence comparison, the GLUT isoforms can be grouped into three classes: class I comprises GLUT14; class II, GLUT6, 8, 10, and 12 and class III, GLUT5, 7, 9, 11 and HMIT. Despite their sequence similarity and the presence of class-specific signature sequences, these transporters carry various hexoses and HMIT is a $\mathrm{H}^{+}$/ myo-inositol co-transporter. Furthermore, the substrate transported by some isoforms has not yet been identified. Tissue- and cell-specific expression of the well-characterized GLUT isoforms underlies their specific role in the control of whole-body glucose homeostasis. Numerous studies with transgenic or knockout mice indeed support an important role for these transporters in the control of glucose utilization, glucose storage and glucose sensing. Much remains to be learned about the transport functions of the recently discovered isoforms (GLUT6-13 and HMIT) and their physiological role in the metabolism of glucose, myo-inositol and perhaps other substrates.
\end{abstract}

Keywords Facilitative diffusion - Glucose transporter . GLUT · Glucose metabolism · Diabetes

\section{Introduction}

Facilitated diffusion of glucose and related hexoses across biological membranes is catalysed by members of the SLC2 family, referred to as glucose transporters or

M. Uldry · B. Thorens $(\bullet)$

Institute of Pharmacology and Toxicology,

University of Lausanne,

27, Rue du Bugnon, 1005 Lausanne, Switzerland

e-mail: Bernard.Thorens@ipharm.unil.ch
GLUTs. These transporters function as simple carriers and the movement of hexose across the plasma membrane proceeds in the direction imposed by its electrochemical gradient. A common structural feature of the SLC2 family member is the presence of 12 transmembrane domains (TM) with both the amino and carboxy-terminal ends present on the cytosolic side and a unique $N$-linked oligosaccharide side-chain present either in the first or the fourth extracellular loop. Signature sequences conserved between the different members of the SLC2 family are present at distinct locations in the primary structure (Fig. 2). The presence of these sequences, however, does not predict the substrate specificity of these transporters.

Glucose transporters are expressed in every cell of the body, as might be anticipated from the key role of glucose in providing metabolic energy and building blocks for the synthesis of biomolecules. The specific physiological role of the isoforms expressed in tissues involved in the control of glucose homeostasis, i.e. muscle, adipose tissue, liver, pancreatic $\beta$-cells and brain, has been studied in greatest detail. Indeed, in these tissues glucose transporters play important roles in the control of glucose utilization, glucose production and glucose sensing and their dysregulated expression may underlie pathogenetic mechanisms leading to development of diabetes mellitus, but also other specific monogenic diseases (see below).

Facilitated diffusion of glucose across plasma membranes has been studied for several decades [43]. The recognition that human erythrocytes have a high density of glucose transporters allowed the initial biochemical purification of this transporter and the preparation of specific antibodies. These were then used for initial cloning of a human glucose transporter by screening an expression library prepared from a human hepatoma cell line (HepG2) [53]. This glucose transporter, GLUT1, was then used for subsequent cloning, by low-stringency screening, of GLUT2-5. More recently, novel glucose transporter-related proteins have been identified by screening gene databases for sequences similar to consensus sequences derived from GLUT1-5. These novel molecules are now known as GLUT6-12 and HMIT. 


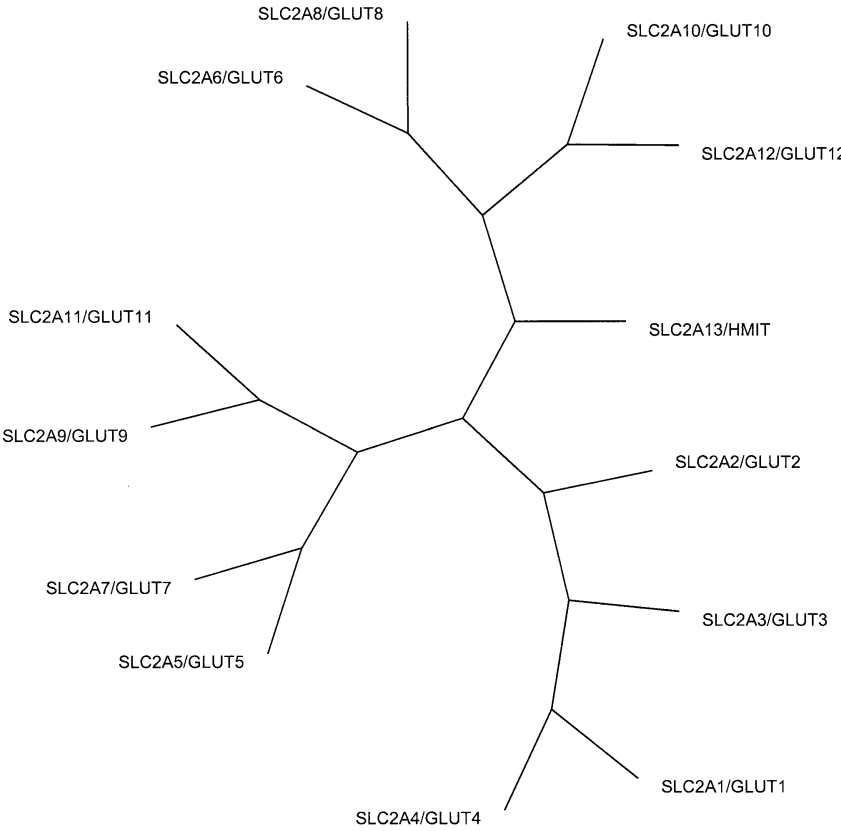

Fig. 1 Radial tree showing multiple alignment of all members of the extended glucose transporter (GLUT) family and constructed using the clustalW program from the EMBL European Bioinformatics Institute (http://www.ebi.ac.uk/clustalw/). The three subclasses of the family are clearly distinguishable (class 1: GLUT1-4; class 2: GLUT5, 7, 9 and 11; class 3: GLUT6, 8, 10, 12 and the $\mathrm{H}^{+}$/ myo-inositol transporter HMIT)

Figure 1 shows a dendrogram of the glucose transporter family and the global arrangement of the molecules in the plasma membrane. Structurally, these molecules can be divided in three classes: GLUT1-4 (class 1); GLUT5, 7, 9 and 11 (class 2) and GLUT6, 8, 10, 12 and HMIT (class $3)$. GLUT1-4 are the initially characterized glucose transporters. GLUT5 is a fructose transporter and the function of the other transporter-like molecule from class 2 is not yet firmly established. The class- 2 proteins lack the tryptophan equivalent to W388 of GLUT1. Among the class-3 family, the function of GLUT8 and 10 as glucose transporters has been established clearly, and HMIT is an $\mathrm{H}^{+} /$myo-inositol symporter. The function of GLUT6 and 12 is not yet defined. One particular structural characteristic of the class-3 molecules is the short extracellular loop between TM1 and -2 and a longer loop between TM10 and -11 that contains the unique $N$ glycosylation site.

\section{GLUT1}

Glut 1 was the first transporter to be characterized by molecular cloning, and its cDNA was isolated from an expression library using antibodies against the humans erythrocyte glucose transporter [53]. Although cloned from a hepatoma cDNA library, GLUT1 is not expressed in normal hepatocytes. It is, however, induced during oncogenic transformation of most cell types and its expression correlates with the increase in glucose metabolism observed in tumour cells [20]. GLUT1 is found in almost every tissue with different levels of expression in different cell types. The expression level usually correlates with the rate of cellular glucose metabolism. It is also expressed highly in blood-tissue barriers, in particular in the endothelial cells forming the blood-brain barrier [45].

The topological arrangement of GLUT1 within the plasma membrane has been confirmed using several experimental approaches. Recently, two models have been proposed for the tertiary structure of GLUT1. The first is based on data obtained from cysteine scanning mutagenesis of five of the $\alpha$-helices of GLUT1 together with information from site-directed mutagenesis [52]. The second is based primarily on the proposed helical bundle arrangement of the Lac permease and has been refined using energy minimization algorithm [79]. These two models describe a key role for helix 7 in the formation of a water-filled channel which may form the path for glucose across the plasma membrane.

The transport of glucose may be described as an alternating conformer model in which the transporter has mutually exclusive binding sites located on the extracellular (import site) and on the intracellular face (export site) of the transporter. Binding of glucose to one site induces the transporter to switch to the opposite conformation, a process that is accompanied by a movement of the substrate across the plasma membrane. In human erythrocytes, GLUT1 is thought to be present as homodimers or homotetramers, with the conversion between both oligomeric forms being dependent on the redox state $[27,28]$. GLUT1 transports glucose with a $K_{\mathrm{m}}$ of $\sim 3 \mathrm{mM}$. Other transported substrates are galactose, mannose and glucosamine [75].

Glucose transport by GLUT1 is sensitive to several inhibitors that also block transport by other isoforms. Many of them are competitive inhibitors of sugar binding, either to the extracellular or the cytosolic sugar binding sites. Cytochalasin B binds to the inner surface of GLUT1 [4] and inhibits its glucose transport activity with an $\mathrm{IC}_{50}$ of $0.44 \mu \mathrm{M}$. Binding of cytochalasin B is to a site which contains tryptophan 388 and 412 (see Fig. 2). Also acting on the same intracellular site is the diterpene toxin forskolin. Forskolin has been used as a photoaffinity label with some specificity for the glucose transporter and its affinity is increased in the 3-iodo4-azidophenethylamido7-O-succinyldeacetyl (IAPS) derivative. An iodinated derivative of forskolin (7-aminoalkylcarbamate) with a very high affinity $\left(\mathrm{IC}_{50} 200 \mathrm{nM}\right)$ has also been described [51].

Glucose transport activity of GLUT1 is inhibited by $\mathrm{HgCl}_{2}\left(\mathrm{IC}_{50} 3.5 \mu \mathrm{M}\right)$, phloretin $\left(\mathrm{IC}_{50} 49 \mu \mathrm{M}\right)$ phlorizin $\left(\mathrm{IC}_{50} 355 \mu \mathrm{M}\right)$ [37] and 4,6-O-ethylidene-D-glucose ( $\mathrm{IC}_{50}$ $12 \mathrm{mM}$ ), which binds to the external glucose binding site where glutamine 161 appears to be critical for inhibitor binding [54]

Several heterozygous mutations resulting in GLUT1 haploinsufficiency have been identified. These cause 
Fig. 2 Schematic model of the structure of classes 1 and 2 (upper panel) and class 3 (lower panel) members of the GLUT family. The signature sequences of the GLUT family are highlighted. The tryptophan residues 388 and 412 (numbering of GLUT1) are part of the cytochalasin B binding site. The major differences between classes 1 and 2 and class 3 are the position of the large extracellular loop containing the $\mathrm{N}$ glycosylation site(s), the proline-containing motif between transmembrane domains (TM) TM6 and TM7, and the presence of a dileucine motif in the amino-terminal tail of class-3 transporters (except for GLUT10)
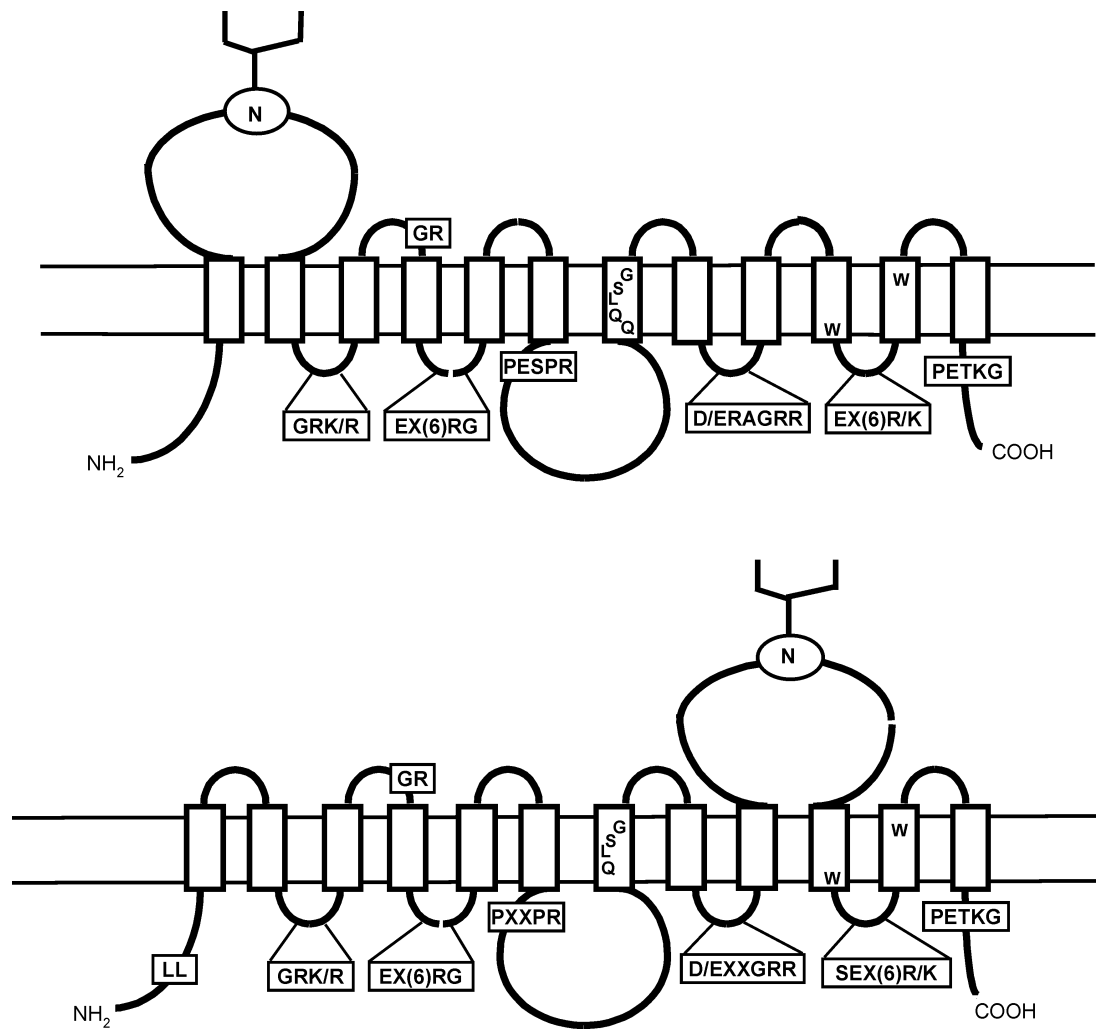

hypoglycorrachia, a condition characterized by seizures, developmental delay, acquired microcephaly, and hypotonia, and which is due to a decrease rate of glucose transport from the blood into cerebrospinal fluid $[42,67]$.

\section{GLUT2}

GLUT2 cDNA was cloned by low-stringency screening of rat [72] and human [21] liver cDNA libraries with a GLUT1 cDNA probe. GLUT2 is a low-affinity transporter for glucose $\left(K_{\mathrm{m}} \sim 17 \mathrm{mM}\right)$ [34], galactose $\left(K_{\mathrm{m}}\right.$ $\sim 92 \mathrm{mM})$, mannose $\left(K_{\mathrm{m}} \sim 125 \mathrm{mM}\right)$ and fructose $\left(K_{\mathrm{m}}\right.$ $\sim 76 \mathrm{mM}$ ), but a high-affinity transporter for glucosamine $(\sim 0.8 \mathrm{mM})$ [75]. The capacity for transporting fructose, shared also by GLUT5 and GLUT8, could be explained by the absence of the QLS motif from helix 7 (residues 279-281 of mouse GLUT1). Indeed, mutation of these amino acids in GLUT3 confers fructose transport capability on this transporter [66]. Absence of this motif could also be responsible of the surprisingly high affinity of GLUT2 for glucosamine.

GLUT2 binds cytochalasin B with a fivefold lower affinity ( $\left.\mathrm{IC}_{50} 1.9 \mu \mathrm{M}\right)$ than GLUT1 [34] and the exofacial reagent 2- $N$-4-(1-azi-2,2,2-trifluoroethyl)benzoyl-1,3bis(D-mannose-4-yloxy)-2-propylamine (ATB-BMPA) with the same affinity as GLUT1 or GLUT4 (IC 50 $0.3 \mathrm{mM}$ ). GLUT2 is also inhibited by phloretin and phlorizin.

GLUT2 is present in the basolateral membrane of intestine and kidney absorptive epithelial cells [73]. In these cells it participates with the apically located, $\mathrm{Na}^{+}-$ dependent glucose transporters SGLT1 (SLC5A1) and SGLT2 (SLC5A2) [76] in the transepithelial transport of glucose (Fig. 3). In hepatocytes, GLUT2 is present in the sinuosidal membrane where it is involved in both glucose uptake and glucose release into the blood. GLUT2 is present at high levels in the plasma membrane of pancreatic $\beta$-cells where it catalyses the first step in glucose-stimulated insulin secretion, a glucose metabolism-dependent signalling mechanism where phosphorylation of glucose by glucokinase represents the ratecontrolling step (Fig. 3) [25, 48]. Functional studies have also demonstrated the requirement for GLUT2 in other glucose-sensing units, in particular those in the hepatoportal vein [12], the hypothalamus and brain stem. These glucose-sensing units are involved in the control of counter-regulation, food intake and stimulation of glucose uptake by peripheral tissues [11].

Mice in which the GLUT2 gene has been inactivated display early diabetes due to impaired glucose-stimulated insulin secretion and abnormal postnatal pancreatic islet development [23]. The early death of the animals is due to the suppression of glucose-stimulated insulin secretion. Reexpression of GLUT2 by viral transduction of isolated islets restores normal insulin secretion and transgenic reexpression of GLUT2 in the pancreatic $\beta$-cells of GLUT2-null mice also restores the normal stimulation by glucose of insulin secretion and allows mouse survival and breeding [25]. These mice with the rescued $\beta$-cell phenotype, however, show suppressed function of the hepatoportal glucose sensors that control peripheral 

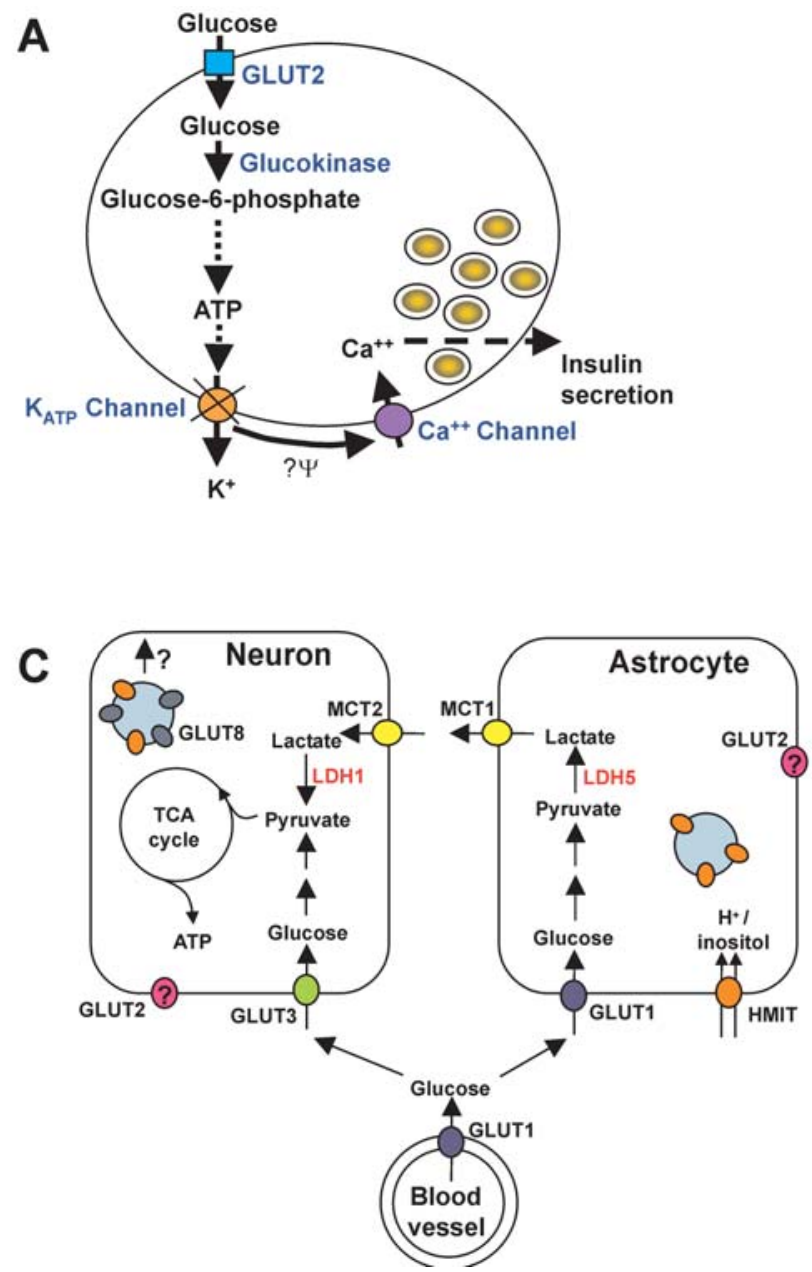

Fig. 3 A Glucose transporter and glucose sensing. Pancreatic $\beta$ cells secrete insulin in response to elevations in the blood glucose concentration. The signalling pathway controlling insulin secretion is based on glucose metabolism and the production of factors coupling metabolism to depolarization of the plasma membrane. This is followed by opening of $\mathrm{Ca}^{++}$channels and the rise in intracellular $\mathrm{Ca}^{++}$leads to exocytosis of the insulin granule. The rate-controlling step in this signalling pathway is the phosphorylation of glucose by glucokinase. Glucose uptake is catalysed by GLUT2, which allows rapid equilibration of glucose between the extracellular medium and the cell's cytoplasm. Suppression of GLUT2 by gene targeting prevents normal access of glucose to glucokinase and thus impairs glucose-stimulated insulin secretion. Physiological studies in GLUT2 $2^{-/-}$mice led to the identification of GLUT2-dependent, extrapancreatic glucose sensors controlling glucose utilization in peripheral tissues, counter-regulation and food intake (see text). B Glucose uptake in insulin-sensitive tissues. In muscle and fat, glucose uptake is stimulated by insulin. This mechanism involves activation of insulin receptor signalling cascades to stimulate translocation of GLUT4-containing vesicles from an intracellular site towards, and fusion with, the plasma membrane. An increased number of GLUT4 at the cell surface increases the rate of glucose uptake, a rate-limiting step in glucose metabolism in these tissues. Following termination of insulin signalling, GLUT4 is re-internalized through clathrin-coated pits and becomes available for a subsequent round of insulin-triggered cell surface exposure. C Brain and blood-brain barrier transporters. Transport of glucose into the brain requires, first, translocation through the endothelial cells forming the blood-brain barrier. GLUT1 is the major isoform present in both the luminal and

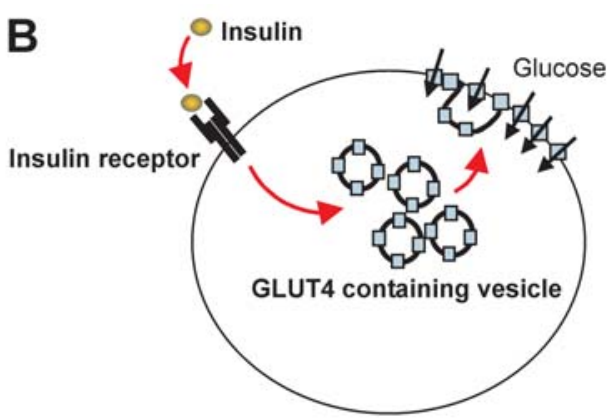

Muscle cell or adipocyte
D Intestine

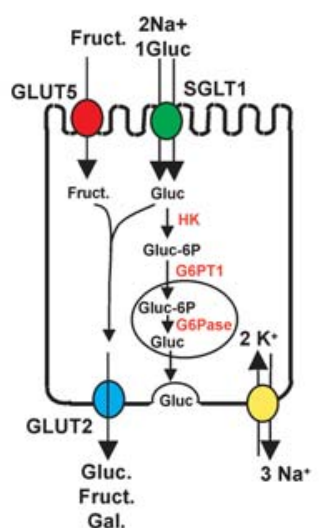

Renal Proximal convolutated Tubule
Renal Proximal straight Tubule abluminal plasma membranes of these cells. Glucose enters astrocytes through GLUT1. In astrocytes, glucose is catabolized to lactate, which may be delivered to neurons through the monocarboxylate transporters $M C T 1$ and $M C T 2$. In neurons, lactate is converted to pyruvate (via lactate dehydrogenase $L D H$ ) that enters the tricarboxylic acid (TCA) cycle to generate ATP. Glucose can also be taken up by neurons through GLUT3. A subset of neurons also express GLUT8. Whereas GLUT3 is present mostly on the plasma membrane, GLUT8 is mostly intracellular and its cell surface expression probably depends on a translocation mechanism not yet elucidated. GLUT2 is expressed in the brain but its precise localization is under investigation. HMIT is present at relatively high level in both astrocytes and neurons. It is involved in $\mathrm{H}^{+}-$ dependent myo-inositol uptake in both cell types. D Transepithelial hexose absorption and reabsorption. In the intestine, transepithelial glucose transport is initiated by glucose and galactose concentrative transport through the $\mathrm{Na}^{+}$-dependent glucose transporter SGLT1 and fructose by facilitated diffusion through GLUT5. These hexoses can all exit the basolateral membrane through GLUT2. This requires glucose phosphorylation (via hexokinase $H K$ ), entry of glucose-6-phosphate (Gluc-6P) into the endoplasmic reticulum via the Gluc-6P transporter-1 (G6PT1), followed by hydrolysis by glucose-6-phosphatase (G6Pase) and release into the extracellular space by a membrane-traffic-based mechanism. A similar mechanism also operates in hepatocytes for glucose release. In the kidney proximal tubule, where $\sim 80 \%$ of the glucose is reabsorbed, transepithelial transport is initiated at the apical membrane by SGLT2 and GLUT2 is the basolateral glucose transporter. In the proximal straight tubule, the remaining glucose is reabsorbed using the apical SGLT1 and GLUT1 in the basolateral membrane 
glucose utilization, and of the central sensors that control glucagon secretion and food intake. Interestingly, glucose production rate by the liver and isolated hepatocytes is not impaired in the absence of GLUT2 although glucose uptake is suppressed. This indicates the presence of a transport mechanism for glucose output independent of facilitated diffusion across the plasma membrane and probably relying on membrane traffic-based mechanisms. $[24,31]$. Similarly, transepithelial glucose transport in the intestine is unimpaired in the absence of GLUT2 and may also rely on a membrane traffic pathway [70].

In human, inactivating mutations of the GLUT2 gene underlie the Fanconi-Bickel syndrome [63]. This is a rare, autosomal recessive disorder of carbohydrate metabolism characterized by hepatorenal glycogen accumulation, tubular nephropathy, glucose and galactose intolerance and fasting hypoglycaemia. As in GLUT2-null mice, intestinal uptake of glucose still proceeds normally and glucose output from the liver can also be stimulated by glucagon or adrenaline, indicating the presence of alternative mechanisms for glucose transport, also probably relying on membrane traffic mechanisms.

\section{GLUT3}

GLUT3 was cloned from a human fetal muscle cDNA library [40]. GLUT3 transports glucose with high affinity [ $K_{\mathrm{m}} 1.4 \mathrm{mM}$ for 2-deoxy-D-glucose (2-DOG)], and also galactose, mannose, maltose, xylose or dehydroascorbic acid. GLUT3 is inhibited by cytochalasin B with a $K_{\mathrm{i}}$ of $0.4 \mu \mathrm{M}$ and by phloretin and phlorizin [56].

GLUT3 mRNA expression is almost ubiquitous in humans, although the protein distribution is restricted to brain and testis, where it is present in spermatozoa [26], and also to human skeletal muscle, predominantly in the triads of slow-twitch fibres [69]. In mouse and rat, GLUT3 mRNA is detected only in the brain [40]. Immunohistochemical and in situ hybridization analyses have shown GLUT3 to be present in neurons, mainly at the plasma membrane (Fig. 3) although a fraction of the transporter has been found in intracellular vesicles distinct from synaptic vesicles [71]. GLUT3 is also present in the $\alpha$-granules of human platelets, from whence it is translocated to the cell surface in response to thrombin stimulation, a phenomenon that may reflect the platelet's increased energy requirements upon activation [29]. In cultured L6 muscle cells GLUT3 cell surface expression is increased by insulin or insulin growth factor-1 [7].

\section{GLUT4}

GLUT4 was cloned from human [22] rat [8, 14] and mouse tissues [36]. GLUT4 has a $K_{\mathrm{m}}$ for glucose transport of $\sim 5 \mathrm{mM}$. In addition, GLUT4 can also transport dehydroascorbic acid and glucosamine $\left(K_{\mathrm{m}} \sim 3.9 \mathrm{mM}\right)$. Transport is inhibited by cytochalasin $\mathrm{B}\left(\mathrm{IC}_{50} 0.1-\right.$ $0.2 \mu \mathrm{M})$. Glucose transport activity of GLUT4 is inhibited by phloretin $\left(\mathrm{IC}_{50} 10 \mu \mathrm{M}\right)$ and phlorizin $\left(\mathrm{IC}_{50} 140 \mu \mathrm{M}\right)$ [38]. Recently, the protease inhibitor indinavir has been shown to inhibit GLUT4 $\left(\mathrm{IC}_{50} 50 \mu \mathrm{M}\right)$ non-competitively in adipocytes. GLUT4 is the major glucose transporter of brown and white adipose tissue and of skeletal and cardiac muscle. The direct inhibition of GLUT4 transport activity by protease inhibitors may contribute to the insulin resistance observed in patients suffering from acquired immune deficiency syndrome (AIDS) and being treated with indinavir [55].

GLUT4 has two internalization sequences, a dileucine repeat present in the C-terminal tail and a FxxY motif in the amino-terminal end. These motifs are responsible for GLUT4 association with an intracellular tubulo-vesicular compartment in basal low plasma insulin conditions [3]. Binding of insulin to its cell surface receptor leads to a rapid translocation of GLUT4 to the cell surface, resulting in an increase in cellular glucose transport activity (Fig. 3). GLUT4 translocation to the plasma membrane can also be stimulated by exercise. This involves a signalling pathway different from that activated by insulin. This pathway may be regulated by the AMPactivated protein kinase (AMPK). The kinetics of GLUT4 cell surface translocation and endocytosis has been extensively studied using the photoaffinity label ${ }^{3} \mathrm{H}$ ATB-BMPA [30]. This has been applied to the study of GLUT4 recycling in adipocytes and muscles of animals and humans following insulin signalling and exercise training. The ability of insulin to stimulate glucose uptake relies on a complex signalling cascade [62] that is still not understood completely. A defect in the ability of insulin to regulate this metabolic event is one of the key physiological dysfunctions of type- 2 diabetes. A decreased expression of GLUT4 may also cause insulin resistance. However, in type-2 diabetes decreased expression of GLUT4 is observed only in adipose tissue and not in muscle, although the latter is responsible for $\sim 90 \%$ of glucose utilization in the post-prandial state and is thus a major site of insulin resistance.

Mice have been generated with general or tissuespecific inactivation of the GLUT4 gene. Mice heterozygous for a GLUT4-null allele exhibit reduced GLUT4 expression in adipose tissue and skeletal muscle. These mice have increased serum glucose and insulin levels, reduced muscle glucose uptake, hypertension and heart and liver morphological alterations similar to those in humans with type-2 diabetes [68]. Mice with homozygous GLUT4 gene inactivation are very abnormal with short size, enlarged hearts and shorter life-spans. Although normoglycaemic under fasted and fed conditions, they exhibit hyperinsulinaemia in the fed state and impaired insulin tolerance [39].

Tissue-specific disruption of the GLUT4 in muscle results in profound reductions in basal glucose transport and the near absence of stimulation by insulin or contraction. Such mice show severe insulin resistance and glucose intolerance from an early age [78]. Disruption of GLUT4 in adipose tissue results in markedly impaired insulin-stimulated glucose uptake in adipocytes 
and, surprisingly, to insulin resistance in muscle and liver. This may be due to altered secretion by adipocytes of factors that could regulate insulin sensitivity in these other tissues [2]. Selective deletion of GLUT4 from the heart induces modest cardiac hypertrophy associated with increased myocyte size. Basal and isoprotenerol-stimulated isovolumic contractile performance is unaffected [1].

\section{GLUT5}

GLUT5 was isolated from human intestinal epithelial cell [41] and from rat [60] and rabbit [50] jejunum cDNA libraries. When expressed in oocytes, human GLUT5 does not exhibit glucose transport activity [10] but is a fructose transporter $\left(K_{\mathrm{m}} \sim 6 \mathrm{mM}\right)$ and is not inhibited by cytochalasin B, phloretin or phlorizin [47]. In addition to its ability to transport fructose, the rat clone also transports glucose to a lesser extent. Only the glucose transport activity mediated by this isoform is inhibited by cytochalasin B [60].

GLUT5 is expressed primarily in the jejunal region of the small intestine. Its mRNA is also detected at low levels in human kidney, skeletal muscle, and adipocytes. In brain it has been found in microglial cells and in the human blood-brain barrier [46]. GLUT5 plays an important role in fructose absorption by the intestine (Fig. 3). GLUT5 is located mostly in the apical membrane of epithelial cells but has also been reported to be present on the lateral membrane in human intestinal epithelial cells [9].

\section{GLUT6}

The human GLUT6 cDNA (formerly designated GLUT9) was cloned by PCR and rapid amplification of cDNA ends (RACE)-PCR [17] on the basis of sequence information obtained from murine expressed sequence tags (ESTs) and a human genomic sequence. Human GLUT6 mRNA is expressed predominantly in the brain, spleen and peripheral leucocytes but expression of the protein has not yet been demonstrated. When reconstituted in liposomes, GLUT6 transport activity is found only in the presence of $5 \mathrm{mM}$ but not $1 \mathrm{mM}$ substrate and exhibits a low cytochalasin B binding affinity. However, when GLUT6, mutated for its N-terminal dileucine internalization motif, is expressed in Xenopus oocytes, it is expressed at the cell surface but no transport activity for glucose is detected over a wide range of concentrations, nor for fructose, galactose, mannose (M. Ibberson, M. Uldry, B. Thorens, unpublished observations). When transfected in primary adipocytes, GLUT6 is retained in an intracellular compartment. Co-transfecting the cells with a dynamin mutant leads to cell-surface expression of the transporter, indicating a possible recycling of the protein through the plasma membrane. However no stimulus inducing translocation of GLUT6 protein to the plasma membrane has yet been identified.

\section{GLUT7}

GLUT7 was identified in a genome homology search [35]. This gene has not been characterized yet but could be a fructose transporter since it is mostly similar to GLUT5.

\section{GLUT8}

GLUT8 (formerly GLUTX1) was the first isoform of the extended SLC2 family to be identified by database mining and functional expression. Sequences for human, rat and mouse GLUT8 have been identified and cloned by this strategy $[18,32]$.

When expressed in Xenopus oocytes or in mammalian cells, GLUT8 is entirely retained in intracellular compartment. Plasma membrane expression can however be induced by mutating the $\mathrm{N}$-terminal dileucine internalization motif. Measurement of transport activity in oocytes, reveals a relatively high affinity for glucose with a $K_{\mathrm{m}}$ of $\sim 2 \mathrm{mM}$. Fructose and galactose compete with this activity and cytochalasin B inhibits GLUT8. Similar results are obtained when GLUT8 expressed in Cos cells is reconstituted in liposomes.

GLUT8 mRNA is expressed at high level in the testis, at a lower level in the cerebellum, adrenal gland, liver, spleen, brown adipose tissue and lung. In situ hybridization and immunofluorescence detection studies show GLUT8 to be expressed in differentiating spermatocytes of the type- 1 stage in the testis, but to be undetectable in mature spermatozoa [33]. Another study, however, has reported GLUT8 immunoreactivity in the head of mouse and human mature spermatozoa, in the acrosomal region [65]. The basis for these differences is not known but may be related to the different antibodies used. The data of [33] are however in agreement with the in situ hybridization data. In the brain, the protein is found in hippocampal excitatory and inhibitory neurons [13, 33], in dentate gyrus neurons, amygdala and primary olfactory cortex, hypothalamic nuclei and the nucleus of the tractus solitarius. High GLUT8 levels are detected in the supraoptico-hypohyseal tract and immunogold microscopy has shown expression in synaptic vesicles of nerve endings present in the supraoptic nucleus and in the vasopressin-containing secretory granules of the posterior pituitary neurons [33].

GLUT8 is also present in blastocyst, and translocates from an intracellular compartment to the cell surface in response to insulin [13]. GLUT8 may play a crucial role in glucose metabolism of blastocysts since its suppression by antisense oligonucleotides leads to an increased rate of apoptosis [59]. 


\section{GLUT9}

Human GLUT9 cDNA was isolated by PCR amplification on the basis of sequence information from ESTs and from its genomic sequence. To date, no data have been published on the sugar transport activity of the protein. Its highest degree of similarity is with GLUT5, suggesting that it may be a fructose transporter. GLUT9 mRNA is detected almost exclusively in the kidney and liver and at low levels in the small intestine, placenta, lung and leucocytes [58].

\section{GLUT10}

Human and mouse GLUT10 cDNAs were isolated by $3^{\prime}-$ and 5'-RACE-PCR, with sequence information from ESTs and from genomic sequence $[16,49]$. The deduced amino acid sequence presents all sugar transporter signatures but, surprisingly, with the exception of a PESPR motif just after helix 6 that is conserved for all the GLUT isoforms. When expressed in Xenopus oocytes, human GLUT10 exhibits 2-DOG transport with very high affinity $\left(K_{\mathrm{m}}\right.$ $\sim 0.3 \mathrm{mM}$ ). Galactose and glucose compete with 2-DOG uptake and transport is inhibited by phloretin. Absence of the PESPR motif is therefore not critical for the functionality of this transporter and may explain its high affinity for 2-DOG compared with the other GLUTs.

GLUT10 mRNA is detected in the human heart, lung, brain, liver, skeletal muscle, pancreas, placenta and kidney. RT-PCR analysis has also shown GLUT10 mRNA in fetal brain and liver. The subcellular localisation has not yet been studied but may be influenced by the presence of an internalisation motif YSRI at the Cterminal extremity of the transporter. Interestingly the chromosome localization of GLUT10 is in a loci associated with type- 2 diabetes and is thus a possible candidate for a susceptibility gene involved in this disease [16].

\section{GLUT11}

The human GLUT11 cDNA was first isolated by PCR on the basis of sequence information obtained from ESTs and from a genomic sequence [19]. Other groups have identified three different, alternative spliced forms of this gene. This is due to the existence of three different first exons (GLUT11-a, -b and -c) coding for three different Nterminal extremities of 7,14 and 10 amino acids respectively $[64,77]$. Interestingly, exon 1 -c encodes a dileucine motif that may influence the subcellular localisation of GLUT11-c. When GLUT11 transiently expressed in HEK293 cells is reconstituted in liposomes, glucose transport activity is detected and GLUT11 may have low affinity for this substrate. This activity is inhibited by fructose and GLUT11 exhibits low affinity for cytochalasin B [19]. This property has been verified for the splice variants GLUT11-a and -b.
RT-PCR analysis has shown the longer spliced form GLUT11-b to be expressed selectively in the liver, brain, lung and trachea, whereas the shorter GLUT11-a is found in most tissues except the liver. Northern blot analysis, which represents cumulated expression of the three spliced forms, has shown GLUT11 mRNA to be expressed in various tissues, most abundantly in skeletal muscle and heart, at an intermediate level in the brain, small intestine, lung and peripheral blood leukocytes and at low levels in the liver, kidney and placenta. The presence of the protein has been confirmed in heart and skeletal muscle with the help of a C-terminal antibody that recognizes all spliced forms of GLUT11. Immunofluorescence microscopy has revealed plasma membrane expression GLUT11-a when over-expressed in HEK293T cells. In those cells, GLUT11 is a glycoprotein of $42 \mathrm{kDa}$ that can be converted to a $38-\mathrm{kDa}$ polypeptide by peptide $\mathrm{N}$-glycosidase F (PNGase F) digestion [77]. Because of its specific expression in muscle, the protein might participate in the regulation of the glucose homeostasis.

\section{GLUT12}

GLUT12 cDNA was cloned from a human embryonic cDNA library and detected in breast cancer cells [61]. The substrate specificity of the protein is unknown. Specific expression has been found in the heart, skeletal muscle, brown adipose tissue, prostate and in the pregnant and lactating rat mammary gland [44].

\section{HMIT}

Rat and human HMIT (SLC2A13) cDNAs were cloned by screening spleen and frontal cortex cDNA libraries from both species [74]. Its deduced amino acid sequence presents all motifs important for glucose transport activity. Functional expression of HMIT in Xenopus oocytes and in mammalian cells has shown maximal plasma membrane expression after mutation of two internalization motifs and one endoplasmic reticulum (ER) retention signal. Transport activity is specific for myo-insositol and is activated strongly by decreasing the extracellular $\mathrm{pH}$, which increases $V_{\max }$ without changing the $K_{\mathrm{m}}(\sim 100 \mu \mathrm{M})$. Related inositol stereoisomers compete with myo-inositol transport, which is also inhibited by phloretin, phlorizin and cytochalasin B. No glucose transport can be detected. Electrophysiological and cellular acidification measurements have shown that HMIT is a $\mathrm{H}^{+}$-coupled myo-inositol symporter.

The HMIT transcript is expressed predominantly in the brain, with high expression found in hippocampus, hypothalamus, cerebellum and brainstem. A low level of expression has been detected in the white, brown and epididymal adipose tissues and in the kidney. Presence of HMIT protein has been confirmed in the rat brain as a glycoprotein of $75-90 \mathrm{kDa}$ that can be converted to $67 \mathrm{kDa}$ upon enzymatic deglycosylation. HMIT is found in both neurons and glial cells, where it is expressed 
partly at the plasma membrane in vivo, suggesting the possible involvement of a unknown stimulus triggering HMIT translocation to the cell surface. Predominant central expression of HMIT suggests that it has a key role in the control of myo-inositol metabolism in the brain. In the brain, myo-inositol is a major osmolyte and also serves as the precursor for phosphatidylinositol, the major inositol-containing phospholipid. Phosphatidylinositol and inositol polyphosphates are key regulators of various processes taking place at synapse and growth cones, in particular vesicle endo- and exocytosis, polymerisation of microfilaments, regulation of ion channels and signal transduction by Gq-coupled receptors [15].

\section{GLUTs in the CNS and blood brain barrier}

Glucose is the preferred energy substrate of the brain. Due to its expression in the endothelial cells forming the blood brain barrier, GLUT1 is essential for glucose delivery to the brain. Given the fact that the abluminal surface of brain capillaries is covered by specialized astrocytic end-feet that also express GLUT1, the astrocytes probably constitute a major site of glucose uptake. In astrocytes, glucose is catabolized by glycolysis to lactate, which may be delivered to neurons through a glial-specific monocarboxylate transporter (MCT1) and a neuron-specific one (MCT2). In neurons, lactate is converted to pyruvate, which enters the tricarboxylic acid cycle to generate ATP. Glucose can also be taken up directly by neurons, which express the GLUT3 isoform [57]. GLUT2 is also expressed in the brain in specific regions such as the hypothalamus and the brain stem where it may participate in the mechanisms of glucose sensing involved in the control of glucose homeostasis.

The role of GLUT8 in some specific neurons remains unclear. It is localized to intracellular vesicles and may possibly move to the cell surface upon as yet unidentified stimuli [33]. Finally, HMIT is expressed in astrocytes and in neurons. In astrocytes, HMIT is both intracellular and at the plasma membrane, whereas its subcellular localization in neurons is under investigation [74].

\section{Pharmaceutical relevance}

Elevation of blood glucose is the main symptom of types1 or -2 diabetes. The GLUT isoforms that transport glucose represent therefore a potential therapeutic target for normalizing glycaemia. A compound that increases the $V_{\max }$ of GLUT1 would increase whole-body glucose utilization. Given the fact that this isoform is almost ubiquitous, such activation could, however, also lead to severe hypoglycaemia. Another possible site of action for limiting the blood glucose level would be inhibition of glucose absorption in the intestine or reabsorption in the kidney. In the intestine, this could be possible by blocking both GLUT2 and the alternative membrane-traffic-based pathway of basolateral glucose release. In the kidney, GLUT2 deficiency results in glucose excretion in the urine, which decreases glycaemia [23]. Inhibition of GLUT2 specifically in the kidney could thus treat hyperglycaemia. However, SGLT2 seems to be a more interesting target in the kidney for this purpose since its expression is more limited.

Type- 2 diabetes is characterized by the loss of insulin sensitivity that leads to a decrease in GLUT4 translocation to the plasma membrane in response to a high blood glucose. To compensate the resulting reduced flux of glucose into muscle or adipocytes, it would be useful to find a pharmacological compound that increases the $V_{\max }$ of GLUT4 for glucose, or stimulate its translocation to the cell surface.

An impaired brain inositol metabolism has been linked to psychiatric diseases, in particular bipolar disorders. Indeed, current treatments of these mood disorders relies on the use of lithium salts, valproic acid and carbamazepine, drugs whose action may interfere with inositol metabolism. It is well established that one mechanism of action of $\mathrm{Li}^{+}$is the inhibition of inositol monophosphate phosphatase and polyphosphoinositide 1-phosphate phosphatase [6], which blocks recycling of inositol phosphate and reduces the availability of inositol for subsequent cycles of intracellular signal transduction. Inhibition of HMIT could also lead to such beneficial effects for bipolar disorders by decreasing the intracellular inositol concentration.

Some members of the GLUT family (GLUT1, 2 and 4) can transport glucosamine, which is important in the biosynthesis of glycoproteins and, in particular, glycosaminoglycan synthesis in cartilage [75]. In association with collagen fibres, these molecules are responsible for the resilience of the cartilage to deformation. Destruction of joint cartilage occurs in osteoarthritis, and several studies have shown that glucosamine is beneficial for this disease. Given the fact that GLUT1 is expressed in chondrocytes, the cells that synthesize cartilage, glucosamine's favourable effects for osteoarthritis are probably mediated by transport across GLUT1 into these cells. Furthermore, glucosamine absorption seems to be mediated in part by GLUT2. This provides an example of the use of GLUT isoforms to deliver therapeutic molecules to their site of action.

\section{References}

1. Abel ED, Kaulbach HC, Tian R, Hopkins JC, Duffy J, Doetschman T, Minnemann T, Boers ME, Hadro E, ObersteBerghaus C, Quist W, Lowell BB, Ingwall JS, Kahn BB (1999) Cardiac hypertrophy with preserved contractile function after selective deletion of GLUT4 from the heart. J Clin Invest 104:1703-1714

2. Abel ED, Peroni O, Kim JK, Kim YB, Boss O, Hadro E, Minnemann T, Shulman GI, Kahn BB (2001) Adipose-selective targeting of the GLUT4 gene impairs insulin action in muscle and liver. Nature 409:729-733

3. Al-Hasani H, Kunamneni RK, Dawson K, Hinck CS, MullerWieland D, Cushman SW (2002) Roles of the N- and C-termini of GLUT4 in endocytosis. J Cell Sci 115:131-140

4. Baldwin SA, Lienhard GE (1989) Purification and reconstitution of glucose transporter from human erythrocytes. Methods Enzymol 174:39-50 


\section{Deleted}

6. Berridge MJ, Downes CP, Hanley MR (1989) Neural and developmental actions of lithium: a unifying hypothesis. Cell 59:411-419

7. Bilan PJ, Mitsumoto Y, Maher F, Simpson IA, Klip A (1992) Detection of the GLUT3 facilitative glucose transporter in rat L6 muscle cells: regulation by cellular differentiation, insulin and insulin-like growth factor-I. Biochem Biophys Res Commun 186:1129-1137

8. Birnbaum MJ (1989) Identification of a novel gene encoding an insulin-responsive glucose transporter protein. Cell 57:305-315

9. Blakemore SJ, Aledo JC, James J, Campbell FC, Lucocq JM, Hundal HS (1995) The GLUT5 hexose transporter is also localized to the basolateral membrane of the human jejunum. Biochem J 309:7-12

10. Burant CF, Takeda J, Brot-Laroche E, Bell GI, Davidson NO (1992) Fructose transporter in human spermatozoa and small intestine is GLUT5. J Biol Chem 267:14523-14526

11. Burcelin R, Thorens B (2001) Evidence that extrapancreatic GLUT2-dependent glucose sensors control glucagon secretion. Diabetes 50:1282-1289

12. Burcelin R, Dolci W, Thorens B (2000) Glucose sensing by the hepatoportal sensor is GLUT2-dependent: in vivo analysis in GLUT2-null mice. Diabetes 49:1643-1648

13. Carayannopoulos MO, Chi MM, Cui Y, Pingsterhaus JM, McKnight RA, Mueckler M, Devaskar SU, Moley KH (2000) GLUT8 is a glucose transporter responsible for insulin-stimulated glucose uptake in the blastocyst. Proc Natl Acad Sci USA 97:7313-7318

14. Charron MJ, Brosius FC 3rd, Alper SL, Lodish HF (1989) A glucose transport protein expressed predominately in insulinresponsive tissues. Proc Natl Acad Sci USA 86:2535-2539

15. Cremona O, De Camilli P (2001) Phosphoinositides in membrane traffic at the synapse. J Cell Sci 114:1041-1052

16. Dawson PA, Mychaleckyj JC, Fossey SC, Mihic SJ, Craddock AL, Bowden DW (2001) Sequence and functional analysis of GLUT10: a glucose transporter in the Type 2 diabetes-linked region of chromosome 20q12-13.1. Mol Genet Metab 74:186-199

17. Doege H, Bocianski A, Joost HG, Schurmann A (2000) Activity and genomic organization of human glucose transporter 9 (GLUT9), a novel member of the family of sugartransport facilitators predominantly expressed in brain and leucocytes. Biochem J 350:771-776

18. Doege H, Schurmann A, Bahrenberg G, Brauers A, Joost HG (2000) GLUT8, a novel member of the sugar transport facilitator family with glucose transport activity. J Biol Chem 275:16275-16280

19. Doege H, Bocianski A, Scheepers A, Axer H, Eckel J, Joost HG, Schurmann A (2001) Characterization of human glucose transporter (GLUT) 11 (encoded by SLC2A11), a novel sugartransport facilitator specifically expressed in heart and skeletal muscle. Biochem J 359:443-449

20. Flier JS, Mueckler MM, Usher P, Lodish HF (1987) Elevated levels of glucose transport and transporter messenger RNA are induced by ras or src oncogenes. Science 235:1492-1495

21. Fukumoto H, Seino S, Imura H, Seino Y, Eddy RL, Fukushima Y, Byers MG, Shows TB, Bell GI (1988) Sequence, tissue distribution, and chromosomal localization of mRNA encoding a human glucose transporter-like protein. Proc Natl Acad Sci USA 85:5434-5438

22. Fukumoto H, Kayano T, Buse JB, Edwards Y, Pilch PF, Bell GI, Seino S (1989) Cloning and characterization of the major insulin-responsive glucose transporter expressed in human skeletal muscle and other insulin-responsive tissues. J Biol Chem 264:7776-7779

23. Guillam MT, Hummler E, Schaerer E, Yeh JI, Birnbaum MJ, Beermann F, Schmidt A, Deriaz N, Thorens B, Wu JY (1997) Early diabetes and abnormal postnatal pancreatic islet development in mice lacking Glut-2. Nat Genet 17:327-330

24. Guillam MT, Burcelin R, Thorens B (1998) Normal hepatic glucose production in the absence of GLUT2 reveals an alternative pathway for glucose release from hepatocytes. Proc Natl Acad Sci USA 95:12317-12321

25. Guillam MT, Dupraz P, Thorens B (2000) Glucose uptake, utilization, and signaling in GLUT2-null islets. Diabetes 49:1485-1491

26. Haber RS, Weinstein SP, O’Boyle E, Morgello S (1993) Tissue distribution of the human GLUT3 glucose transporter. Endocrinology 132:2538-2543

27. Hamill S, Cloherty EK, Carruthers A (1999) The human erythrocyte sugar transporter presents two sugar import sites. Biochemistry 38:16974-16983

28. Hebert DN, Carruthers A (1992) Glucose transporter oligomeric structure determines transporter function. Reversible redoxdependent interconversions of tetrameric and dimeric GLUT1. J Biol Chem 267:23829-23838

29. Heijnen HF, Oorschot V, Sixma JJ, Slot JW, James DE (1997) Thrombin stimulates glucose transport in human platelets via the translocation of the glucose transporter GLUT-3 from alpha-granules to the cell surface. J Cell Biol 138:323-330

30. Holman GD, Kozka IJ, Clark AE, Flower CJ, Saltis J, Habberfield AD, Simpson IA, Cushman SW (1990) Cell surface labeling of glucose transporter isoform GLUT4 by bis-mannose photolabel. Correlation with stimulation of glucose transport in rat adipose cells by insulin and phorbol ester. J Biol Chem 265:18172-18179

31. Hosokawa M, Thorens B (2002) Glucose release from GLUT2null hepatocytes: characterization of a major and a minor pathway. Am J Physiol 282:E794-E801

32. Ibberson M, Uldry M, Thorens B (2000) GLUTX1, a novel mammalian glucose transporter expressed in the central nervous system and insulin-sensitive tissues. J Biol Chem 275:4607-4612

33. Ibberson M, Riederer BM, Uldry M, Guhl B, Roth J, Thorens B (2002) Immunolocalization of GLUTX1 in the testis and to specific brain areas and vasopressin-containing neurons. Endocrinology 143:276-284

34. Johnson JH, Newgard CB, Milburn JL, Lodish HF, Thorens B (1990) The high $K_{\mathrm{m}}$ glucose transporter of islets of Langerhans is functionally similar to the low affinity transporter of liver and has an identical primary sequence. J Biol Chem 265:6548-6551

35. Joost HG, Thorens B (2001) The extended GLUT-family of sugar/polyol transport facilitators: nomenclature, sequence characteristics, and potential function of its novel members (review). Mol Membr Biol 18:247-256

36. Kaestner KH, Christy RJ, McLenithan JC, Braiterman LT, Cornelius P, Pekala PH, Lane MD (1989) Sequence, tissue distribution, and differential expression of mRNA for a putative insulin-responsive glucose transporter in mouse 3T3-L1 adipocytes. Proc Natl Acad Sci USA 86:3150-3154

37. Kasahara T, Kasahara M (1996) Expression of the rat GLUT1 glucose transporter in the yeast Saccharomyces cerevisiae. Biochem J 315:177-182

38. Kasahara T, Kasahara M (1997) Characterization of rat Glut4 glucose transporter expressed in the yeast Saccharomyces cerevisiae: comparison with Glut1 glucose transporter. Biochim Biophys Acta 1324:111-119

39. Katz EB, Stenbit AE, Hatton K, DePinho R, Charron MJ (1995) Cardiac and adipose tissue abnormalities but not diabetes in mice deficient in GLUT4. Nature 377:151-155

40. Kayano T, Fukumoto H, Eddy RL, Fan YS, Byers MG, Shows TB, Bell GI (1988) Evidence for a family of human glucose transporter-like proteins. Sequence and gene localization of a protein expressed in fetal skeletal muscle and other tissues. J Biol Chem 263:15245-15248

41. Kayano T, Burant CF, Fukumoto H, Gould GW, Fan YS, Eddy RL, Byers MG, Shows TB, Seino S, Bell GI (1990) Human facilitative glucose transporters. Isolation, functional characterization, and gene localization of cDNAs encoding an isoform (GLUT5) expressed in small intestine, kidney, muscle, and adipose tissue and an unusual glucose transporter pseudogenelike sequence (GLUT6). J Biol Chem 265:13276-13282 
42. Klepper J, Voit T (2002) Facilitated glucose transporter protein type 1 (GLUT1) deficiency syndrome: impaired glucose transport into brain - a review. Eur J Pediatr 161:295-304

43. Lieb WR, Stein WD (1971) New theory for glucose transport across membranes. Nat New Biol 230:108-109

44. Macheda ML, Williams ED, Best JD, Wlodek ME, Rogers S (2003) Expression and localisation of GLUT1 and GLUT12 glucose transporters in the pregnant and lactating rat mammary gland. Cell Tissue Res 311:91-97

45. Maher F, Vannucci SJ, Simpson IA (1994) Glucose transporter proteins in brain. FASEB J 8:1003-1011

46. Mantych GJ, James DE, Devaskar SU (1993) Jejunal/kidney glucose transporter isoform (Glut-5) is expressed in the human blood-brain barrier. Endocrinology 132:35-40

47. Mate A, de la Hermosa MA, Barfull A, Planas JM, Vazquez CM (2001) Characterization of D-fructose transport by rat kidney brush-border membrane vesicles: changes in hypertensive rats. Cell Mol Life Sci 58:1961-1967

48. Matschinsky FM (1996) Banting Lecture 1995. A lesson in metabolic regulation inspired by the glucokinase glucose sensor paradigm. Diabetes 45:223-241

49. McVie-Wylie AJ, Lamson DR, Chen YT (2001) Molecular cloning of a novel member of the GLUT family of transporters, SLC2a10 (GLUT10), localized on chromosome 20q13.1: a candidate gene for NIDDM susceptibility. Genomics 72:113117

50. Miyamoto K, Tatsumi S, Morimoto A, Minami H, Yamamoto H, Sone K, Taketani Y, Nakabou Y, Oka T, Takeda E (1994) Characterization of the rabbit intestinal fructose transporter (GLUT5). Biochem J 303:877-883

51. Morris DI, Robbins JD, Ruoho AE, Sutkowski EM, Seamon KB (1991) Forskolin photoaffinity labels with specificity for adenylyl cyclase and the glucose transporter. J Biol Chem 266:13377-13384

52. Mueckler M, Makepeace C (2002) Analysis of transmembrane segment 10 of the Glut1 glucose transporter by cysteinescanning mutagenesis and substituted cysteine accessibility. J Biol Chem 277:3498-3503

53. Mueckler M, Caruso C, Baldwin SA, Panico M, Blench I, Morris HR, Allard WJ, Lienhard GE, Lodish HF (1985) Sequence and structure of a human glucose transporter. Science 229:941-945

54. Mueckler M, Weng W, Kruse M (1994) Glutamine 161 of Glut1 glucose transporter is critical for transport activity and exofacial ligand binding. J Biol Chem 269:20533-20538

55. Murata H, Hruz PW, Mueckler M (2002) Indinavir inhibits the glucose transporter isoform Glut4 at physiologic concentrations. Aids 16:859-863

56. Nelson JA, Falk RE (1993) Phloridzin and phloretin inhibition of 2-deoxy-D-glucose uptake by tumor cells in vitro and in vivo. Anticancer Res 13:2293-2299

57. Pellerin L, Bonvento G, Chatton JY, Pierre K, Magistretti PJ (2002) Role of neuron-glia interaction in the regulation of brain glucose utilization. Diabetes Nutr Metab 15:268-273; discussion 273

58. Phay JE, Hussain HB, Moley JF (2000) Cloning and expression analysis of a novel member of the facilitative glucose transporter family, SLC2A9 (GLUT9). Genomics 66:217-220

59. Pinto AB, Carayannopoulos MO, Hoehn A, Dowd L, Moley KH (2002) Glucose transporter 8 expression and translocation are critical for murine blastocyst survival. Biol Reprod 66:1729-1733

60. Rand EB, Depaoli AM, Davidson NO, Bell GI, Burant CF (1993) Sequence, tissue distribution, and functional characterization of the rat fructose transporter GLUT5. Am J Physiol 264:G1169-1176

61. Rogers S, Macheda ML, Docherty SE, Carty MD, Henderson MA, Soeller WC, Gibbs EM, James DE, Best JD (2002) Identification of a novel glucose transporter-like proteinGLUT-12. Am J Physiol 282:E733-E738

62. Saltiel AR, Pessin JE (2002) Insulin signaling pathways in time and space. Trends Cell Biol 12:65-71
63. Santer R, Groth S, Kinner M, Dombrowski A, Berry GT, Brodehl J, Leonard JV, Moses S, Norgren S, Skovby F, Schneppenheim R, Steinmann B, Schaub J (2002) The mutation spectrum of the facilitative glucose transporter gene SLC2A2 (GLUT2) in patients with Fanconi-Bickel syndrome. Hum Genet 110:21-29

64. Sasaki T, Minoshima S, Shiohama A, Shintani A, Shimizu A, Asakawa S, Kawasaki K, Shimizu N (2001) Molecular cloning of a member of the facilitative glucose transporter gene family GLUT11 (SLC2A11) and identification of transcription variants. Biochem Biophys Res Commun 289:1218-1224

65. Schurmann A, Axer H, Scheepers A, Doege H, Joost HG (2002) The glucose transport facilitator GLUT8 is predominantly associated with the acrosomal region of mature spermatozoa. Cell Tissue Res 307:237-242

66. Seatter MJ, De la Rue SA, Porter LM, Gould GW (1998) QLS motif in transmembrane helix VII of the glucose transporter family interacts with the $\mathrm{C}-1$ position ofD-glucose and is involved in substrate selection at the exofacial binding site. Biochemistry 37:1322-1326

67. Seidner G, Alvarez MG, Yeh JI, O'Driscoll KR, Klepper J, Stump TS, Wang D, Spinner NB, Birnbaum MJ, De Vivo DC (1998) GLUT-1 deficiency syndrome caused by haploinsufficiency of the blood-brain barrier hexose carrier. Nat Genet 18:188-191

68. Stenbit AE, Tsao TS, Li J, Burcelin R, Geenen DL, Factor SM, Houseknecht K, Katz EB, Charron MJ (1997) GLUT4 heterozygous knockout mice develop muscle insulin resistance and diabetes. Nat Med 3:1096-1101

69. Stuart CA, Wen G, Jiang J (1999) GLUT3 protein and mRNA in autopsy muscle specimens. Metabolism 48:876-880

70. Stumpel F, Burcelin R, Jungermann K, Thorens B (2001) Normal kinetics of intestinal glucose absorption in the absence of GLUT2: evidence for a transport pathway requiring glucose phosphorylation and transfer into the endoplasmic reticulum. Proc Natl Acad Sci USA 98:11330-11335

71. Thoidis G, Kupriyanova T, Cunningham JM, Chen P, Cadel S, Foulon T, Cohen P, Fine RE, Kandror KV (1999) Glucose transporter Glut3 is targeted to secretory vesicles in neurons and PC12 cells. J Biol Chem 274:14062-14066

72. Thorens B, Sarkar HK, Kaback HR, Lodish HF (1988) Cloning and functional expression in bacteria of a novel glucose transporter present in liver, intestine, kidney, and beta-pancreatic islet cells. Cell 55:281-290

73. Thorens B, Cheng ZQ, Brown D, Lodish HF (1990) Liver glucose transporter: a basolateral protein in hepatocytes and intestine and kidney cells. Am J Physiol 259:C279-C285

74. Uldry M, Ibberson M, Horisberger JD, Chatton JY, Riederer BM, Thorens B (2001) Identification of a mammalian $\mathrm{H}^{+}$-myoinositol symporter expressed predominantly in the brain. EMBO J 20:4467-4477

75. Uldry M, Ibberson M, Hosokawa M, Thorens B (2002) GLUT2 is a high affinity glucosamine transporter. FEBS Lett 524:199_ 203

76. Wright EM, Turk E (2003) The sodium glucose cotransporter family SLC5. Pflugers Archives, in press (this issue)

77. Wu X, Li W, Sharma V, Godzik A, Freeze HH (2002) Cloning and characterization of glucose transporter 11, a novel sugar transporter that is alternatively spliced in various tissues. Mol Genet Metab 76:37-45

78. Zisman A, Peroni OD, Abel ED, Michael MD, Mauvais-Jarvis F, Lowell BB, Wojtaszewski JF, Hirshman MF, Virkamaki A, Goodyear LJ, Kahn CR, Kahn BB (2000) Targeted disruption of the glucose transporter 4 selectively in muscle causes insulin resistance and glucose intolerance. Nat Med 6:924-928

79. Zuniga FA, Shi G, Haller JF, Rubashkin A, Flynn DR, Iserovich P, Fischbarg J (2001) A three-dimensional model of the human facilitative glucose transporter Glut1. J Biol Chem 276:44970-44975 